\title{
REPRESENTATIVENESS OF OFFENSIVE SCENARIOS TO EVALUATE PERCEPTUAL-COGNIIIIVE SKILLS OF WATER POLO PLAYERS
}

\author{
Filipe Casanova, 1, 2, A, B, C, D, E Ricardo Pereira,, B Sofia Canossa, 1, A, B Maickel Padilha, 1, B \\ Rafael Bagatin, 1, B Israel Teoldo, ${ }^{3, \mathrm{D}}$ Sixto González-Villora, 4, D \\ Ricardo J. Fernandes, ${ }^{1,5, A, D, E}$ Fernando Tavares ${ }^{1, A, E}$ \\ ${ }^{1}$ Centre of Research, Education, Innovation and Intervention in Sport (CIFI2D), Faculty of Sport (FADEUP), University of Porto, Portugal \\ ${ }^{2}$ University Lusófona of Porto, Portugal \\ ${ }^{3}$ Centre of Research and Studies in Soccer, The University Federal of Viçosa, Viçosa, Brazil \\ ${ }^{4}$ Faculty of Education, University of Castilla-La Mancha, Cuenca, Spain \\ ${ }^{5}$ Porto Biomechanics Laboratory (LABIOMEP), University of Porto, Portugal \\ ${ }^{\text {A }}$ Study Design; ${ }^{\mathrm{B}}$ Data Collection; ${ }^{\mathrm{C}}$ Statistical Analysis; ${ }^{\mathrm{D}}$ Manuscript Preparation; ${ }^{\mathrm{E}}$ Funds Collection
}

\author{
Address for corpespondence: \\ Filipe Casanova \\ University of Porto \\ Praça de Gomes Teixeira, 4099-002 Porto, Portugal \\ E-mail: fcasanova@fade.up.pt
}

\begin{abstract}
Ahstract The aim of this study was to trial several attacking offensive sequences as representative for further use in research on the perceptual-cognitive skills of water polo players. Elite water polo coaches were presented with separate test film sequences encompassing 80 structured water polo offensive plays. Each clip was approximately $6 \mathrm{~s}-7 \mathrm{~s}$ long with an inter-clip interval of $5 \mathrm{~s}-10 \mathrm{~s}$, where a red dot was displayed on-screen at the start of the clip to indicate the area of first appearance of the ball. The order of presentation of the video clips was counterbalanced and randomly determined. The criteria were scored on a 5-point Likert-type scale. From the 80 clips presented, only 56 showed high agreement $(W=1 ; p<0.05)$ and internal consistence reliability between the expert observers $(\alpha=0.980 ; p<0.05)$. Furthermore, a very high reproducibility $(Z=0 ; p=1)$ was obtained between viewing sessions. The results obtained determine that 56 offensive scenarios were representative of the water polo game and as such may be useful in evaluating the perceptual-cognitive skills of the players.
\end{abstract}

Key WOrlls decision-making, Attacking Game Scenarios, reliability, Water Polo

\section{Introduction}

Most team sports provide an outstanding stimulating environment where players are required to make accurate and fast decisions due to time, space and opponent constraints (Williams, 2000). In water polo, as in most team sports, along with a proficient execution of motor abilities, perceptual-cognitive skills and tactical knowledge (e.g. declarative knowledge) are of utmost importance to achieve expert performance (Williams, Davids, 1995; Américo et al., 2018). Perceptual-cognitive expertise has been defined as the ability of an individual to locate, 
identify and process environmental information, integrate it with existing knowledge and current motor capabilities, and select and execute appropriate responses (Marteniuk, 1976).

Researchers have identified several perceptive-cognitive skills that could influence sport performance, e.g. pattern recognition (Williams, Hodges, North, Barton, 2006; Williams, Ford, Eccles, Ward, 2011), postural cue usage (Williams, 2002; Savelsbergh et al., 2002; Abernethy, Zawi, 2007), situational probabilities (Williams et al., 2011; Farrow, Reid, 2013) and visual search behaviour (Williams, Davids, Williams, 1999). Moreover, expert players seem to perform more efficiently than novices in a wide range of anticipatory and decisional tasks (Mann, Williams, Ward, Janelle, 2007; Williams, Paul, Julian, Nicolas, 2008). In measuring and understanding the perceptual-cognitive skills underpinning anticipatory skills in sports, a laboratory-based environment (using video or static slides presentations) has mostly been used, due to its inherent experimental control, repeatability and safety (Zeuwts et al., 2016). Those protocols significantly rely in recall and occlusion paradigms that, despite being sensible to detect differences in players expertise level, neglect response times, inhibit correctional perceptive information (static slides) and push reliance on cognitive processes, i.e. video-based trials (Mann et al., 2007).

The abovementioned disadvantages are a direct consequence of a disruption in the sport perception-action cycle, encouraging informational cues and pattern reliance that may not necessarily be used, and employment of different processes in decision making (Abernethy, Katherine, Jerry, 1993; Roca, Williams, Ford, 2014; Zeuwts et al., 2016). These findings are supported by L. Zeuwts et al. (2016) who stated that "the disruption of our natural perception-action cycle might lead to differences in the amount and nature of the information collected, and/or in the way this information is further processed". Concerning the experiment protocol, evidence was found that in goalkeepers, gaze and visual search behaviours function differently, particularly when needing to fix the ball earlier and for a longer during in situ condition with interception vs with movement, and when predicting the penalty kick direction during a video simulation with movement vs with visual reports (Dicks, Button, Davids, 2010).

Despite this lack of environmental validity, video-based trials still prove to be a valid and useful tool in evaluating perceptive-cognitive skills compared to static slides (Casanova, Garganta, Oliveira, 2012), especially when used to distinguish a player's expertise level (North, Ward, Ericsson, Williams, 2011; McRobert, Ward, Eccles, Williams, 2011). For example, it was found that skilled cricket batters were better in anticipation and employed a more refined visual search strategy than less-skilled counterparts using video-based simulations (McRobert et al., 2011).

To accomplish effective video-based perceptual-cognitive skill protocols, firstly, representative and reproducible sport tasks are presented to the athlete, then, the same tasks are used to detect, identify and measure the process-tracing elements through registering visual reports (monitoring eye movements and task manipulations) and, finally, process-tracing element learning is investigated retrospectively and prospectively concerning expertise development (Ericksson, Smith, 1991; Williams, Ericsson, 2005). In addition, to validate representative tasks that could be used to evaluate tactical game understanding, the consensus of six expert level teachers/coaches was collected in the development of a Game Performance Assessment Instrument (GPAl; Oslin, Mitchell, Griffin, 1998). This is a useful and reliable tool to assess players' skill execution and tactical awareness with and without a ball (Santos, Dias, Mendes, Coelho-e-Silva, 2016).

Concerning water polo, few studies have investigated player perceptual-cognitive skills. In a study by E. Kiourmourtzoglou, T. Kourtessis, M. Michalopoulou and V. Derri (1998), the results evidenced that elite water polo players showed better scores than novices on decision-making, visual reaction time and spatial orientation. 
Similarly, L. Quevedo-Junyent, J. Aznar-Casanova, D. Merindano-Encina, G. Cardona and J. Solé-Fortó (2011) found that elite players achieved better dynamic visual acuity scores, and the results also improved for some combinations of speed, contrast and trajectory. Undoubtedly, in water polo, the aquatic medium is a major limitation to implement environmental trials, being a difficult to not only to recreate, but also to use electronic equipment. Therefore, the use of video-based trials seems a reasonable, valid and cost-effectiveness methodology to evaluate perceptual-cognitive skills in water polo players.

As water polo related studies are scare, there is a huge informational gap about the perceptual-cognitive skills underpinning anticipation ability and their relative importance in achieving success.

The main purpose of the current study then was to identify offensive scenarios that could be representative in water polo for assessing the levels of the perceptual-cognitive skills underpinning the anticipatory abilities of players. To accomplish that aim, habilitated and experienced water polo coaches were invited to comment on the representativeness of selected offensive scenarios.

\section{Method}

\section{Participants}

The representativeness of selected offensive scenarios was determined by a panel of 10 licenced water polo coaches with post-graduate Physical Education qualifications and at least 10 years of active training experience. The study was approved by the Ethics Committee of the host University (protocol number CEFADE.02.2019) and all procedures conducted in accordance with the Declaration of Helsinki (1996) norms. Coaches volunteered to participate and filled in an informed consent form.

\section{Match scenarios}

The coach panel watched 80 match clips from the final game of a national mens water polo cup match. The clips randomly represented different offensive game situations, in both even play and temporary numerical superiority. To support identical match characteristics, a shot clock with the official remaining attack time was superimposed in the middle of the clip, but avoiding conflicting visual perception of the ball's flying trajectory (Figure 1). Each trial was filmed from both $10 \mathrm{~m}$ behind and $5 \mathrm{~m}$ above the goal with two cameras (Rollei® 5S FHD, $1920 \times 1080$ pixels, $60 \mathrm{~Hz}$ framerate and $175^{\circ}$ capture angle). The elevated filming position allowed presentation of some elements of visual depth.

Windows Movie Maker® and Wondershare Filmora ${ }^{\circledR}$ version 7.8.0 software were used to edit the game recordings into 80 clips. After display of the clip number, a red dot would appear on-screen marking the starting point of the ball, with the screen turning black when the offensive scenario had finished. Each clip lasted $6 \mathrm{~s}-7 \mathrm{~s}$ and the questionnaires were filled in during the $5 \mathrm{~s}$ to $10 \mathrm{~s}$ intervals between clips. To define clip representativeness, coaches viewed the entire clip, inclusively of the outcome of the play, i.e., shot to goal, shot to goalkeeper defence or out, defensive steal, defensive block, pass to centre-forward with, or not, exclusion of defensive centre-back or offensive foul. 


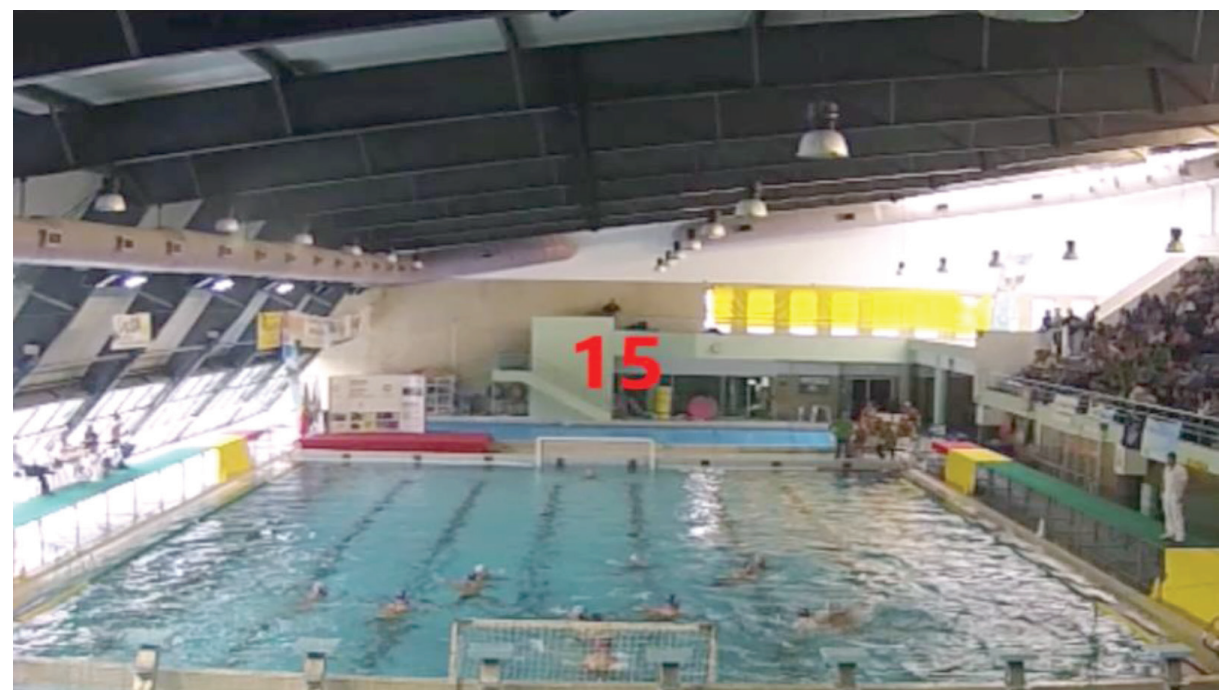

Figure 1. Frame of one offensive scenario presented in the video

\section{Procedures}

All the clip evaluations were conducted in a classroom at the host University, with scenarios being projected (model VPL-EW 255, maximum resolution 1,600 × 1,200 pixels with 3.200 ANSI Lumens of brightness and a contrast rate of $2700: 1)$ from a laptop onto a white screen $(2 \times 3 \mathrm{~m})$. The clip representativeness was scored on a 5-point Likert-type scale questionnaire, in which 1 means "total disagreement" and 5 "total agreement" with the representativeness of the observed offensive pattern. Likert-scales have proven to be valid and reliable psychometric scale to measure attitudes and is commonly used in social studies and marketing research (Hartley, MacLean Jr., 2006; Dawes, 2008), and have been used in perceptual-cognitive expertise in sport related research (Williams et al, 2006). The clip presentation was counterbalanced and randomly determined, during both evaluation meetings (one month between them). To familiarize coaches with the scenarios and the protocol, two additional clips were presented prior to the main assessments.

\section{Data analysis}

Descriptive statistical analysis was used to examine the valid values of the 5-point Likert-type scale, and Kendall's coefficient of concordance (W) was applied to test agreement between the observers. Cronbach's Alpha (a) was used to test the internal consistency reliability between the observers, and nonparametric Wilcoxon Signed Rank tests (Z) allowed verification of the construct validity (observers re-test). The Statistical Package for Social Sciences v 24.0 (SPSS Inc., Chicago, II, USA) was used in all statistical procedures, and a statistical significance of $p<0.05$ was set at for all tests. 


\section{Resulls}

From the 80 water polo clips viewed, 24 were excluded when one or more coaches expressed "total disagreement" or "disagreement" with that specific offensive event. Therefore, a total of 56 clips were accepted for ongoing analysis, with the representativeness of the offensive game events being concordant among the observers in all the clips accepted $(W=1, p<0.05)$. The internal consistency reliability between the observers was maintained $(a=0.980 ; p<0.05)$. Finally, regarding the construct validity of the clips, when the expert coaches watched the scenarios for a second time, the values of the 5 -point Likert-type scale were strongly reproduced $(Z=0 ; p=1$; Table 1).

Table 1. Mean valid values of the 5-point Likert-type scale $( \pm S D)$ pointed by the coaches, in both moments of evaluation

\begin{tabular}{ccc}
\hline Coach & Test & Re-Test \\
\hline 1 & $4.98 \pm 0.134$ & $4.98 \pm 0.134$ \\
2 & $4.98 \pm 0.134$ & $4.98 \pm 0.134$ \\
3 & $4.98 \pm 0.134$ & $4.98 \pm 0.134$ \\
4 & $4.96 \pm 0.187$ & $4.96 \pm 0.187$ \\
5 & $4.98 \pm 0.134$ & $4.98 \pm 0.134$ \\
6 & $4.98 \pm 0.134$ & $4.98 \pm 0.134$ \\
7 & $4.98 \pm 0.134$ & $4.98 \pm 0.134$ \\
8 & $4.96 \pm 0.187$ & $4.96 \pm 0.187$ \\
9 & $4.98 \pm 0.134$ & $4.98 \pm 0.134$ \\
10 & $4.98 \pm 0.134$ & $4.98 \pm 0.134$ \\
\hline
\end{tabular}

\section{Discussion}

Accepting that offensive clips are a useful tool to evaluate perceptual-cognitive skills under controlled laboratory trials (Williams et al., 1999), the main purpose of the current study was to establish representative water polo offensive scenarios for further use in perceptual-cognitive skills research. A panel of 10 expert coaches agreed that 56 clips were representative of the water polo game and, since the clips were taken from a major national competition final, all game characteristics were validated. It should be noted that player decisions in the clips would not always be the most appropriate in the offensive plays (as happens in any other sport).

In water polo, four main game phases are considered: counterattack, even play, transition, and power play. Since the transition phase was not addressed in this study, and no counterattack occurred, all the remaining game phases were retracted in the observed scenarios. In closed matches (up to a three goal difference between the winning and losing teams) of a water polo world championship, it was observed that the team's ability to gain exclusion fouls and score during temporary numerical superiority (power play) defined the winner and the loser (Lupo, Tessitore, Minganti, Capranica, 2010). These authors also stated that the centre-forward position had a determinant role, so the most direct offensive solution is to unbalance the opposition's defensive game play and then make a pass to the centre-forward. Moreover, a tendency was found for higher level teams to finish an even play situation with a centre-forward action, rather than perimeter players obtaining a power play situation or a penalty (Lupo et al., 2012). Moreover, E. Mirvic, F. Rasidagic, N. Nurkovic, H. Kajmovic, C. Lupo (2019) reported on 
the outcomes of the Brazilian Olympic Games women's water polo matches, and noted that close and unbalanced games were decided by non-specific plays, and only a few by technical and tactical factors.

Power play and counterattack efficiency had been considered determinant in defining winning and losing teams when the match is close or unbalanced (Canossa, Garganta, Argudo, Fernandes, 2009; Saavedra, Escalante, Madera, Mansilla, Garcia-Hermoso, 2014). Corroborating the above mentioned observations, in evaluating 88 games from the Spanish Professional Water polo League to identify offensive performance indicators that best determined a match status (wining, losing or drawing), it was found that the attack outcome most determined match status and, for games with penalties, counterattack shots and counterattacks determined match status (Ordoñez, Pérez, González, 2016). Another central aspect of the game is goalkeeper efficiency, considered determinant for winning/losing outcomes in major national competitions (Escalante et al., 2011; Saavedra et al., 2014). Accordingly, a good strategy may lie in fatiguing and misplacing the goalkeeper, opening opportunities to efficiently score.

The use of video scenarios in evaluating such a conclusion would allow video editing to occlude, speed up, slow down, add informational or irrelevant cues, tied with other measurements (e.g. eye movement registration and verbal reports) to allow a global understanding of the players' perceptual-cognitive skills in several decision-making tasks (Casanova et al., 2012), promoting a better understanding of the main discriminate variables of successful and unsuccessful water polo teams. In addition, video-based trials can be very useful due to their repeatability, safety and experimental control (Abernethy et al., 1993; Ali, 2011; Vickers, 2007; Casanova et al., 2012). Concerning further perceptual-cognitive expertise evaluations, the clip duration was maintained between $6 \mathrm{~s}$ and $7 \mathrm{~s}$ as athletes can accurately recall complete sequences after a 0.5 s-10 s task (Eriksson, Simon, 1999).

In conjunction with scenes, temporal occlusion has been used in some studies for decision-making tests in which players were required to anticipate the near future action that was executed at the end of each video clip (Williams et al., 1994; Casanova et al., 2013). The methodological technique used to occlude the scene (i.e. information) at a specific time in a critical event of information extraction by players (e.g. before the moment that the player will touch the ball and will continue the action), allows the researchers to evaluate player abilities to recognize and use de postural cues, so differencing expertise across skill groups (Williams et al., 2011).

Some caution is required when developing task designs for evaluating perceptual-cognitive skills, namely in the control of variables, the outcomes and test validity, for understanding functional human behaviour (Causer, Barach, Williams, 2014). Some research limitations should be pointed out too, as the level of Portuguese Water polo is below many countries that have good results in European and World competitions. Despite no study being conducted to assess the differences in declarative knowledge between water polo players and coaches in Portugal and other countries, these differences likely exist. The players would also be evaluated in a context with no physical fitness or fatigue, so it is difficult to determine how such differences in cognition and gaze control affect performance.

Nevertheless, and as stated above, this framework allows a great experimental control, repeatability and safety control (Abernethy et al., 1993; Ali, 2011; Vickers, 2007; Casanova et al., 2012). Furthermore, the representative offensive clips defined in this study may open a bridge for a major understanding of the water polo game and distinguish expert from non-expert water polo players.

This study suggested that 56 offensive scenarios were indeed representative of the water polo game and as such may be useful for ongoing evaluation of perceptual-cognitive skills of water polo players. Moreover, this powerful and useful tool may allow water polo coaches to analyse and evaluate the anticipatory ability of their 
athletes in a control environment. In addition, and of utmost importance is the understanding of parallelism between laboratory results and real game performance in a water polo match.

\section{Acknowledgements}

This study was part of the INEX project and supported by the Centre of Research, Education, Innovation and Intervention in Sport (CIFI'2D) of the Faculty of Sport (FADEUP) of the University of Porto, Portugal, under postdoctoral research activity.

\section{Author contributions statement}

This research is based on data collected for postdoctoral work by FC. FT and RJF assisted with A. RP, SC, MP and RB assisted with B. IT, SG-V and RJF assisted with D.

\section{Conflict of interest statement}

The authors declare that the research was conducted in the absence of any commercial or financial relationships that could be construed as a potential conflict of interest.

\section{References}

Abernethy, B., Katherine, T., Jerry, T. (1993). Strategies for improving understanding of motor expertise (Or mistakes we have made and things we have learning!!!). In: J. Starkes, F. Allard (eds), Cognitive Issues in Motor Expertise (pp. 317-353). Amsterdam, Netherlands: Elsevier.

Abernethy, B., Zawi, K. (2007). Pickup of essential kinematics underpins expert perception of movement patterns. Journal of Motor Behavior, 39 (5), 353-367.

Ali, A. (2011). Measuring Soccer Skill Performance: A Review. Scandinavian Journal of Medicine and Science Sports, 1-15.

Américo, H.B., Kowalski, M., Cardoso, F., Kunrath, C.A., González-Víllora, S., Teoldo, I. (2018). Difference in declarative tactical knowledge between u-11 and u-15 soccer players. Human Movement, 18 (5), 25-30.

Canossa, S., Garganta, J., Argudo, F., Fernandes, R. (2009). Indicadores táctico-técnicos de sucesso do jogo de polo aquático de elite. Brazilian Journal of Biomotricity, 3 (3), 209-219.

Casanova, F., Garganta, J., Oliveira, J., (2012). Representativeness of offensive scenarios to evaluate perceptual-cognitive expertise of soccer players. The Open Sports Sciences Journal, 5, 161-166.

Casanova, F., Garganta, J., Silva, G., Alves, A., Oliveira, J., Williams, M. (2013). The effects of prolonged intermittent exercise on perceptual-cognitive processes in soccer players. Medicine and Science in Sports and Exercise, 45 (8), 1610-1617.

Causer, J., Barach, P., Williams, M. (2014). Expertise in medicine: using the expert performance approach to improve simulation training. Medical Education, 48 (2), 115-123.

Dawes, J. (2008). Do data characteristics change according to the number of scale points used? An experiment using 5-point, 7-point and 10-point scales. International Journal of Marketing Research, 50 (1), 61-77.

Dicks, M., Button, C., Davids, K. (2010). Examination of gaze behaviors under in situ and video simulation task constraints reveals differences in information pickup for perception and action. Attention, Perception and Psychophysics, 72 (3), 706-720.

Ericsson, K., Smith, J. (1991). Prospects and limits of the empirical study of expertise: An introduction. In: K. Ericsson, J. Smith (eds), Toward a general theory of expertise: Prospects and limits (pp. 1-18). New York: Cambridge University Press.

Eriksson, K., Simon, H. (1999). Protocol Analysis: Verbal reports as data. United States of America: Massachusets Institute of Technology.

Escalante, Y., Saavedra, J., Mansilla, M., Tella, V. (2011). Discriminatory Power of Water Polo Game-related statistics at the 2008 Olympic games. Journal of Sports Sciences, 29 (3), 291-298.

Farrow, D., Reid, M. (2013). Contribuition of Situation Probability Information to Anticipatory Skill. Journal of Science and Medicine in Sport, 15 (4), 368-373. 
Hartley, S., MacLean Jr, W. (2006). A review of the reliability and validity of Likert-type scales for people with intellectual disability. Journal of Intellectual Disability, 50 (part II), 813-827.

Kiourmourtzoglou, E., Kourtessis, T., Michalopoulou, M., Derri, V. (1998). Differences in several perceptual abilities between experts and novices in basketball, volleyball and water-polo. Perceptual and Motor Skills, 86 (3), 899-912.

Lupo, C., Capranica, L., Mingatti, C., Cortis, C., Tessitore, A., Perroni, F. (2012). Effects of competition level on the centre forward role of men's Water Polo. Journal of Sports Sciences, 30 (9), 889-897.

Lupo, C., Tessitore, A., Minganti, C., Capranica, L. (2010). Notational analysis of elite and sub-elite water polo matches. Journal of Strenght and Conditioning Research, 24 (1), 223-229.

Mann, D., Williams, M., Ward, P., Janelle, C. (2007). Perceptual-Cognitive Expertise in Sport: A Meta-Analysis. Journal of Sport and Exercise Psychology, 29, 457-478.

Marteniuk, R.G. (1976). Information processing in motor skills. New York: Holt, Rinehart \& Winston.

McRobert, A., Ward, P., Eccles, D., Williams, M. (2011). The effect of manipulating context-specific information on perceptual-cognitive processes during a simulated anticipation tas. Brithish Journal of Psychology, 102, 519-534.

Mirvic, E., Rasidagic, F., Nurkovic, N., Kajmovic, H., Lupo, C. (2019). Only unbalanced games are affected by technical and tactical aspects able to discriminate winning and losing performance in women's water polo Olympic Games. Sport Science and Health, $15,427-434$.

North, J., Ward, P., Ericsson, A., Williams, M. (2011). Mechanisms underlying skilled anticipation and recognition in a dynamic and temporally constrained domain. Memory, 19 (2), 155-168.

Ordóñez, E.G., Pérez, C.I., González, C.T. (2016). Performance assessment in water polo using compositional data analysis. Journal of Human Kinetics, 54 (1), 143-151.

Oslin, J., Mitchell, S., Griffin, L. (1998). The Game Performance Assessment Instrument (GPAI): Development and Preliminary Validation. Journal of Teaching in Physical Education, 17, 231-243.

Quevedo-Junyent, L., Aznar-Casanova, J., Merindano-Encina, D., Cardona, G., Solé-Fortó, J. (2011). Comparison of Dynamic Visual Acuity Between Water Polo Players and Sedentary Students. Research Quarterly for Exercise and Sport, 82 (4), 644-651.

Roca, A., Williams, M., Ford, P. (2014). Caturing and Testing perceptual-cognitive expertise: A comparison of stationary and movement response methods. Behavioral Research, 46, 173-177.

Saavedra, J., Escalante , Y., Madera, J., Mansilla, M., Garcia-Hermoso, A. (2014). Comparison of Game-Related Statistics in Men's International Champioship's between Winning and Losing Teams according to Margin of Victory. Collegium Antropologicum, 38 (3), 901-907.

Savelsbergh, G.J.P., Williams, M., Van der Kamp, J., Ward, P. (2002). Visual search, anticipation and expertise in soccer goalkeepers. Journal of Sports Sciences, 20, 279-287.

Santos, R., Dias, G., Mendes, R., Coelho-e-Silva, M. (2016). Performance evaluation in sports games: GPAl e TSAP. Conexões: Ciência e Tecnologia, 14 (2), 137-157.

Vickers, J. (2007). Perception, Cognition and Decision Training: The Quiet Eye in Action (1 ${ }^{\text {st }}$ Ed.). Human Kinetics.

Williams, M. (2000). Perceptual skill in soccer: Implications for talent identification and development. Journal of Sports Sciences, 18, $737-750$.

Williams, M. (2002). Perceptual and Cognitive Expertise in Sport. The Psychologist, 15 (8), 416-417.

Williams, M., Davids, K. (1995). Declarative knowledge in sport: A by-product of experience or a characteristic of expertise? Journal of Sport and Exercise Psychology, 17 (3), 259-275.

Williams, M., Davids, K., Burwitz, L., Williams, J.G. (1994). Visual search strategies in experienced and inexperienced soccer players. Research Quarterly for Exercise and Sport, 65 (2), 127-135.

Williams, M., Ericsson, K. (2005). Perceptual-cognitive expertise in sport: Some considerations when applying the expert performance approach. Human Movement Sciences, 24, 283-307.

Williams, M., Davids, K., Williams, J. (1999). Visual Perception and Action in Sports. London: E \& FN Spon.

Williams, M., Ford, P., Eccles, D., Ward, P. (2011). Perceptual-Cognitive Expertise in Sport and its Acquisition: Implications for Applied Cognitive Psychology. Applied Cognitive Psychology, 25, 432-442.

Williams, M., Hodges, N., North, J., Barton, G. (2006). Perceiving patterns of play in dynamic sport tasks: investigating the essential information underlying skilled performance. Perception, 35 (3), 317-332. 
Williams, M., Paul, W., Julian, W., Nicolas, S. (2008). Domain Specificity, Task Specificity and Expert Performance. Research Quarterly for Exercise and Sport, 79 (3), 428-433.

Zeuwts, L., Vansteenkiste, P., Deconinck, F., van Maarseveen, M., Savelsbergh, G., Cardon, G., Lenoir, M. (2016). Is gaze behaviour in a laboratory context similar to that in real-life? A study in bicyclists. Transportation Research part F: Traffic Psychology and Behaviour, 43, 131-140.

Cite this anticle as: Casanova, F., Pereira, R., Canossa, S., Padilha, M., Bagatin, R., Teoldo, I., González-Víllora, S., Fernandes, R.J., Tavares, F. (2020). Representativeness of Offensive Scenarios to Evaluate Perceptual-Cognitive Skills of Water Polo Players. Central European Journal of Sport Sciences and Medicine, 1 (29), 11-19. DOI: 10.18276/cej.2020.1-02. 